\title{
Automatic versus manual segmentation of choroidal neovascularization lesions using optical coherence tomography
}

\begin{abstract}
Purpose: To measure and quantify choroidal neovascularization (CNV) lesion size in classic lesions visualized on Spectral Domain-Optical Coherence Tomography (SD-OCT) using automated segmentation analysis and confirm whether further refinement improves the accuracy of disease regression after loading dose Anti-VEGF injections.

Methodology: A retrospective study of 10 macular degeneration patients diagnosed with $\mathrm{CNV}$. Blinded observers, trained to detect changes on OCT, delineated boundaries of CNV lesions using SD-OCT (Topcon 2000) with automated software and then modified segmentation manually before and after loading dose Ranbizumab injections. Automated measurements were then compared to manual segmentation values. Statistical analysis was performed, taking into account inter-observer variability.
\end{abstract}

Volume 6 Issue I - 2017

Correspondence: Ruhella R Hossain, Department of Ophthalmology, East Kent Hospital University Foundation Trust, Kent CTI 3NG, UK, Email ruhella.hossain@gmail.com

Received: November 02, 2016 | Published: January II, 2017

\section{Ruhella R Hossain, Aishath R Moosa, Nishal Patel \\ Department of Ophthalmology, East Kent Hospital University Foundation Trust, UK}

\begin{abstract}
Results: Automatic and manual segmentation measurements are similar in 1) retinal area and fovea center values both pre-and post-injection; and 2) retinal volume post-injection. Retinal volume pre-injection is 1) similar between the two blinded observers; 2) varies between automatic measurements and observers in some patients; and 3) underestimated for all patients in the automatic measurements. Overall, automatic segmentation detects a general reduction in $\mathrm{CNV}$ lesion size following loading dose injections.

Conclusion: SD-OCT automated segmentation software detects CNV lesions and reports size reduction after the third anti-VEGF injection. SD-OCT automatic segmentation using Topcon segmentation analysis in this small cohort is an accurate diagnostic tool to measure $\mathrm{CNV}$ surface area and volume as a biomarker of $\mathrm{CNV}$ regression.
\end{abstract}

Keywords: wet AMD, segmentation software, CNV, OCT, biomarker, subretinal hyper reflective material
Abbreviations: OCT, optical coherence tomography; RPE, retinal pigment epithelium; ILM, internal limiting membrane; nAMD, neovascular age-related macular degeneration; CNV, choroidal neovascularization; FA, fluorescein angiography; Anti-VEGF, antivascular endothelial growth factor; SD-OCT, spectral-domain optical coherence tomography

\section{Introduction}

Optical coherence tomography (OCT) is a non-invasive imaging modality that provides detailed qualitative and quantitative information about the retinal structure. It produces anatomic images of the posterior vitreous, retina, retinal pigment epithelium (RPE) and anterior choroid by recording optical reflectivity and segmenting the different image layers, including the RPE and internal limiting membrane (ILM); and measures the distance between the ILM and RPE. Segmentation software can calculate the area and volume of half-ellipsoid deviations from the normal RPE curvature; and constructs retinal thickness maps at multiple locations. ${ }^{1,2}$

Concurrent pharmacological and imaging technology advancements allowed for improved outcomes in exudative retinal diseases, including neovascular (wet) age-related macular degeneration (nAMD). Historically, diagnosis and classification of choroidal neovascularization (CNV) lesions in nAMD was based on fluorescein angiography (FA) defined morphology of the choroidal membrane (Figure 1) ${ }^{3,4}$ However, the invasive nature of FA poses a dilemma for follow up, making it difficult to individualize injection frequency schedules in response to anti-vascular endothelial growth factor (anti-VEGF) injections, a previously validated therapy for reducing lesion size and restoring vision in nAMD..$^{2,4-12}$

The validation of anti-VEGF therapy for nAMD created the need for non-invasive imaging modalities to monitor patient responses to treatment. Previous studies showed the usefulness of OCT automated segmentation software for monitoring retinal disease in response to therapy. ${ }^{13-15}$ Using OCT, clinicians are able to individualize injection frequencies according to individual patient's visual needs. Although useful, the automated built in segmentation algorithms are known to have errors. Initial OCT segmentation software had a high error rate, partly due to the image-processing techniques and how to handle pathology, thus limiting its diagnostic utility. ${ }^{16-18}$ However, Spectral Domain-OCT (SD-OCT) systems integrated newer technology and improved segmentation software, allowing for improved imageanalysis, including subretinal hyper reflective material (SHRM), and evaluation of pathology. ${ }^{19-22}$ In addition to segmentation software, SD-OCT systems are integrated with analysis software designed to automatically detect deviations from the RPE contour and quantify their surface area and volume..$^{23-29}$ Assuming classic CNV lesions form half-ellipsoid shapes; one can use such tools with automatic segmentation software in serial imaging to monitor nAMD disease progression and response to therapy (Figure 2)..$^{30-32}$ 


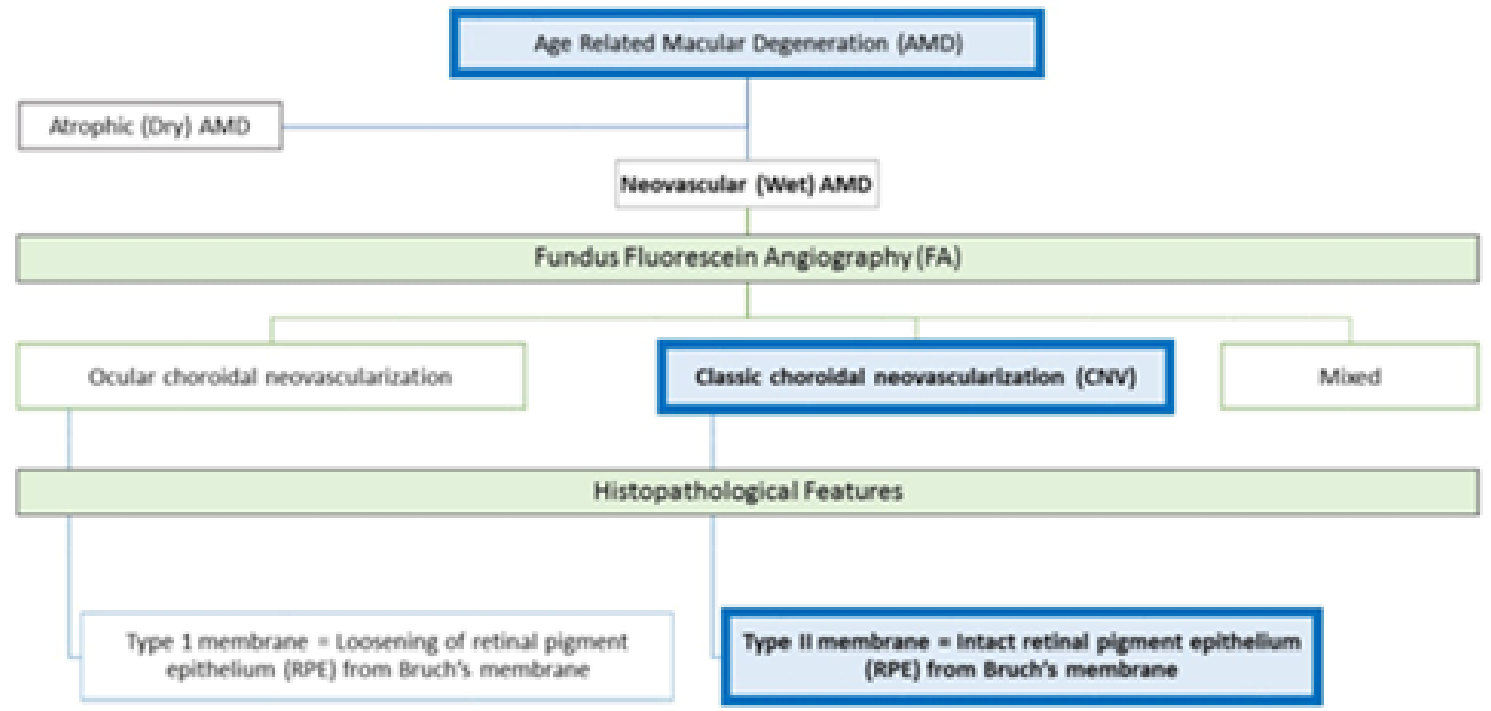

Figure I Classification of age related macular degeneration (AMD).
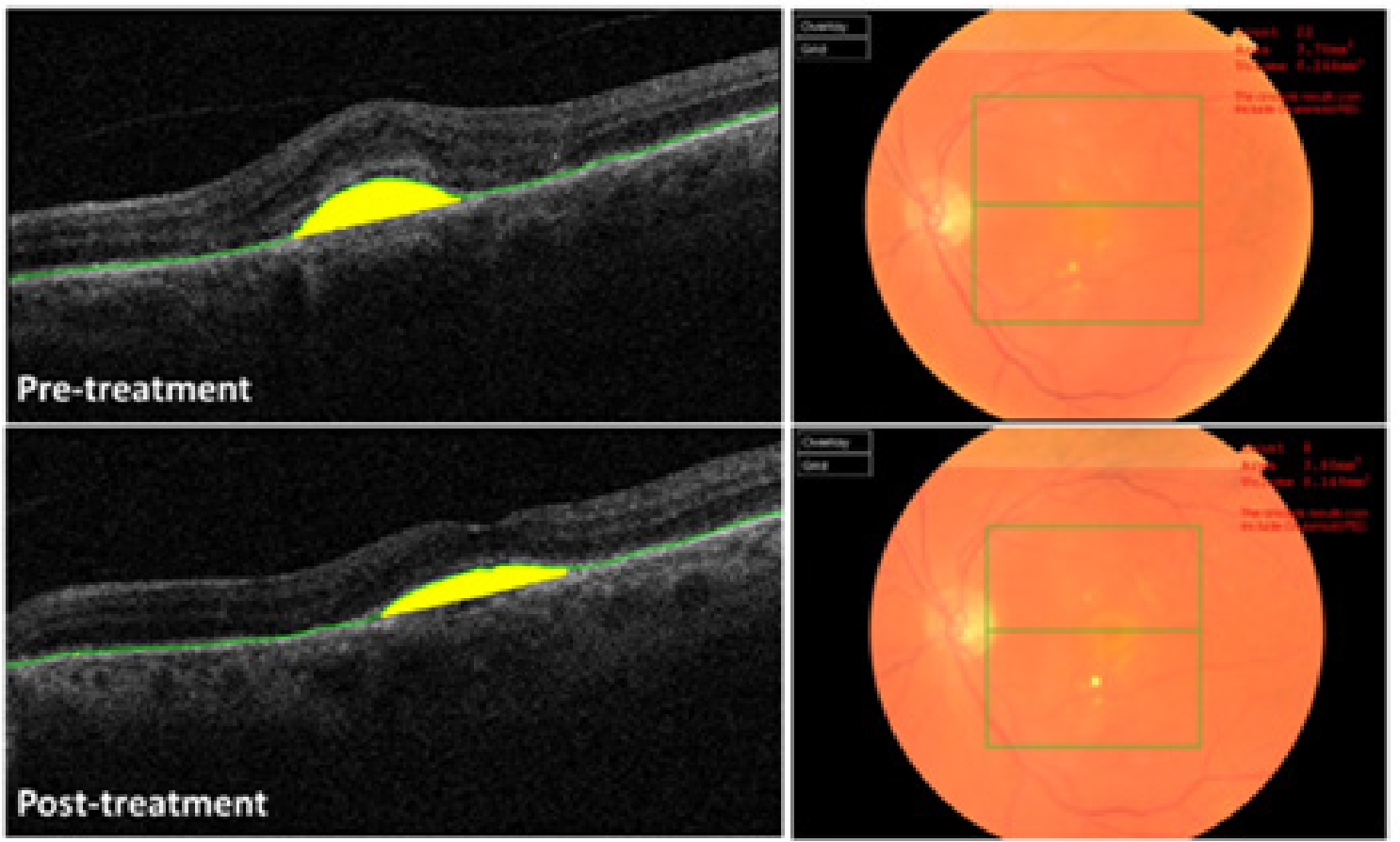

Figure 2 OCT software identification of CNV lesions showing automatic delineation of the RPE and accompanying fundal view A) Pre-treatment and B) Posttreatment.

In this paper we attempt to measure and quantify $\mathrm{CNV}$ lesion size only in classic lesions visualized on SD-OCT using automated drusen analysis and further fine-tune the accuracy to confirm whether optimization can be achieved and whether modification is required. We hypothesize that 1) using surface area and volume as biomarkers for classic CNC lesions, OCT automatic segmentation is as accurate as manual segmentation for delineating lesion borders both pre- and post- anti-VEGF therapy; and 2) OCT automatic segmentation with drusen analysis is an accurate diagnostic tool for measuring classic CNV lesions.

\section{Materials and methods}

This was a retrospective study of 10 AMD patients previously diagnosed with CNV using FA morphology. Two blinded observers, trained to detect RPE changes, manually delineated boundaries of CNV lesions on the inner surface of the RPE using SD-OCT (Topcon 2000) with software analysis before and after three anti-VEGF Lucentis (Ranubizumab) injections. The accompanying segmentation software measured the distance in micrometers between the RPE and Bruch's membrane and automatically calculated the foveal center 
thickness, surface area and volume of the CNV lesion. The generated automatic segmentation measurements were compared against the manual segmented measurements. Statistical analysis was performed using Microsoft Excel and an online statistical calculator, GraphPad (http:/graphpad.com/quickcalcs/kappa1/) for calculating Cohen's kappa score to determine statistical agreement between the automatic segmentation and blinded observers. The average of the two blinded observers was used as the standard. Ethics committee approval was not needed as this study involved a review of data generated from our regular clinical practice. This research followed the tenants of the Declaration of Helsinki.

\section{Results}

SD-OCT serial B-scan images of classic CNV lesions in patients previously diagnosed with nAMD according to FA morphology were assessed for thickness, surface area and volume both pre- and post-treatment. Qualitatively, the automatic segmentation of the RPE closely mimicked that of the manual segmentation in classic CNV lesions. Using RPE segmentation, the SD-OCT software measured foveal center thickness and calculated values for retinal area and volume of the classic $\mathrm{CNV}$ lesions. When comparing the values calculated after automatic segmentation against the two blinded observers we found that the surface area measurements of the CNV lesions were similar both pre- and post-treatment (Figure 3A\&B). Retinal volume measurements varied between automatic and manual pre-injection but were similar post-injection (Figure 3C\&D).

The automatic segmentation underestimated the volume of the CNV lesions for nearly all patients in comparison to manual segmentation. Using the foveal center measurement as a surrogate for CNV lesion thickness, the software calculations for automatic and the two blinded observers were similar both pre- and postinjection (Figure 3E\&F). Despite the quantitative differences between automated and manual segmentations, OCT automatic segmentation detected a general reduction in classic CNV lesion size qualitatively in all 10 patients (Figure 4). Cohen's kappa score was calculated to determine statistical agreement between the automatic and average between the two blinded observers (Table 1). For foveal thickness, we found good and fair strengths of agreement pre- and post-treatment, respectively. For surface area and volume, we found fair and poor strengths of agreement pre- and post-treatment, respectively.
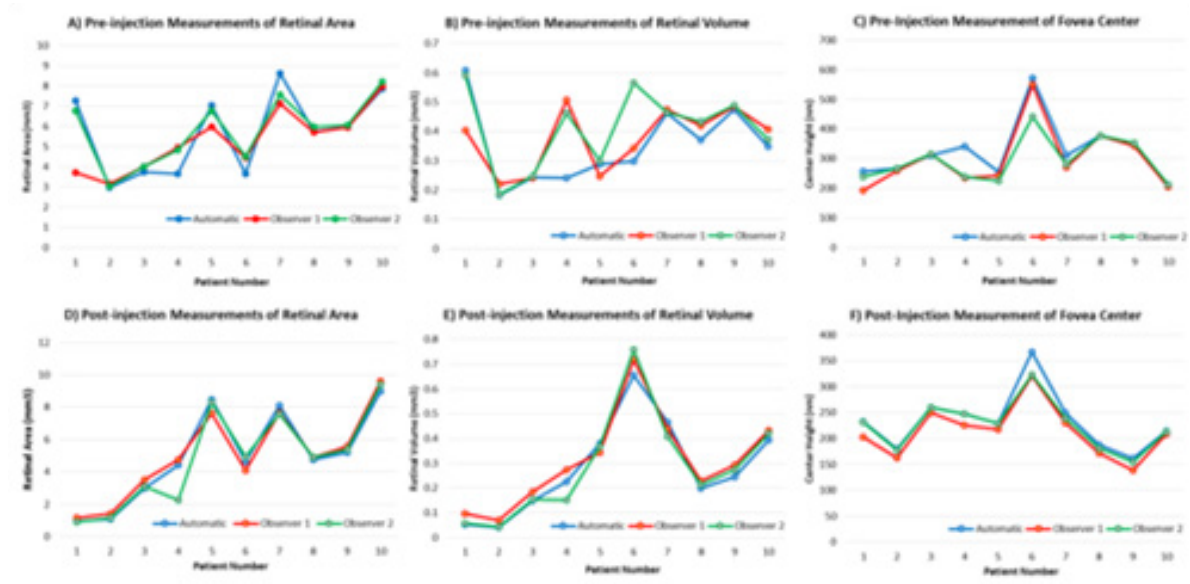

Figure 3 OCT automatic versus manual segmentation of two blinded observers. Pre-treatment measurements of A) Retinal area, B) Retinal volume, C) Foveal center thickness. Post-injection measurements of D) Retinal area, E) Retinal volume, F) Foveal center thickness.

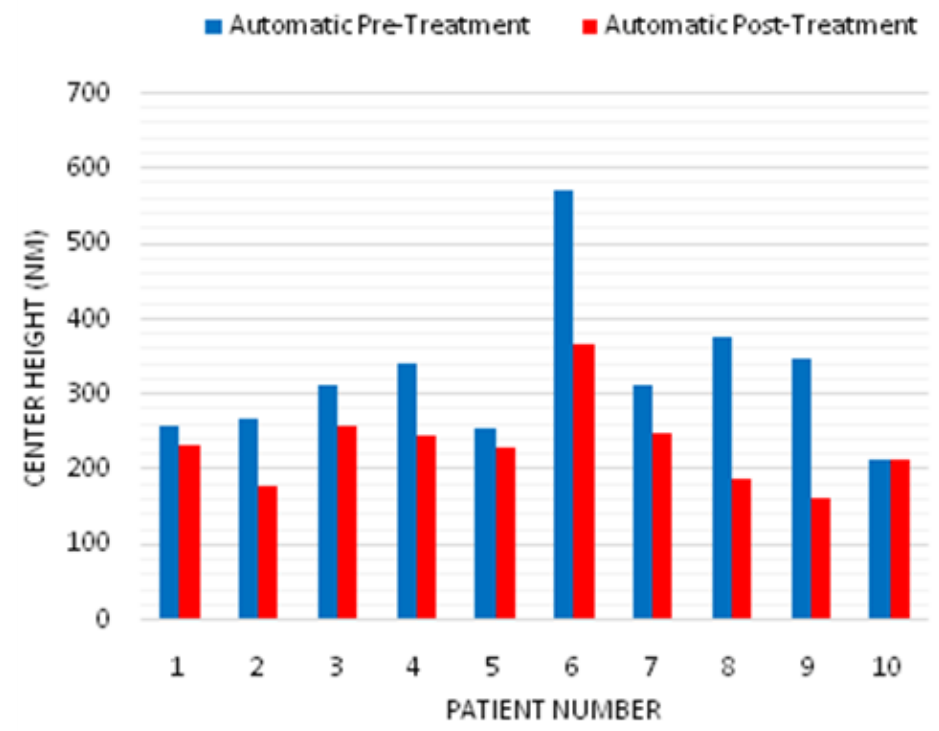

Figure 4 OCT automatic segmentation measurements of foveal thickness pre- and post-treatment. 
Table I Cohen's kappa score for lesion thickness, surface area and volume to determine the statistical agreement between the automatic segmentation and blinded observers, using the average of the two blinded observers as the standard

\begin{tabular}{|c|c|c|}
\hline Thickness & Pre-treatment & Post-treatment \\
\hline k & 0.6 & 0.4 \\
\hline SE of kappa & 0.232 & 0.232 \\
\hline $95 \% \mathrm{Cl}$ & $0.146-1$ & -0.054 to 0.854 \\
\hline$\%$ obs agreed & 80 & 70 \\
\hline $\begin{array}{l}\% \text { agreements expected by } \\
\text { chance alone }\end{array}$ & 50 & 50 \\
\hline Strength of agreement & Good & Fair \\
\hline \multicolumn{3}{|l|}{ Surface Area } \\
\hline k & 0.31 & 0.074 \\
\hline SE of kappa & 0.193 & 0.258 \\
\hline $95 \% \mathrm{Cl}$ & -0.069 to 0.689 & -0.432 to 0.58 I \\
\hline$\%$ obs agreed & 60 & 50 \\
\hline $\begin{array}{l}\% \text { agreements expected by } \\
\text { chance alone }\end{array}$ & 42 & 46 \\
\hline Strength of agreement & Fair & Poor \\
\hline \multicolumn{3}{|l|}{ Volume } \\
\hline k & 4 & 0 \\
\hline SE of kappa & 0.232 & NA \\
\hline $95 \% \mathrm{Cl}$ & -0.054 to 0.854 & NA \\
\hline$\%$ obs agreed & 70 & 90 \\
\hline $\begin{array}{l}\% \text { agreements expected by } \\
\text { chance alone }\end{array}$ & 50 & 90 \\
\hline Strength of agreement & Fair & Poor \\
\hline
\end{tabular}

\section{Discussion}

We used serial SD-OCT B-scan images to monitor the progression of classic CNV lesions in patients both pre- and post-Lucentis treatment, one of the current recommended treatments for nAMD. We used the retinal fovea center thickness, surface area and volume of classic CNV lesions as biomarkers within a group of patients previously diagnosed with nAMD by FA. We compared the results of the automatic segmentation software on the TOPCON SD-OCT machine with that of two blinded observers trained to manually delineate RPE changes.

In our group of patient's we found that the automatic and manual segmentation measurements were similar for 1) fovea center thickness both pre- and post-treatment; 2) retinal area both pre- and post-treatment; and 3) retinal volume post-treatment. Using Cohen's kappa score to determine statistical agreement, we found a good strength of agreement pre-treatment and a fair strength of agreement post-treatment for foveal thickness. With regards to retinal area and volume, we found fair and poor strengths of agreement pre- and posttreatment, respectively. These discrepancies in statistical agreement were expected given that the foveal thickness is a direct measurement whereas the surface area and volume are calculations based on a direct measurement. Thus, the drusen analysis automatic segmentation software is as accurate as manual segmentation when performing direct measurements, not for calculations.

Additionally, we found that pre-injection retinal volume was 1) similar between the two blinded observers; 2) varied between automatic measurement and observers in some patients; and 3) underestimated by the automatic segmentation for all patients in comparison with the manual segmentation. One reason for the underestimation of pre-injection volume by automatic segmentation may be due to the software algorithms used for identifying RPE layers. Although proprietary information, one can assume that these algorithms use an "ideal" physiologic RPE line and follow the curvature of Bruch's membrane. It is possible that any deviation from these ideal examples can lead to over- or under-estimation of volume in comparison to manual segmentation. However, as long as the algorithms are consistent then these over- and under-estimations should not impact the software clinical use for monitoring disease progression.

SD-OCT can be used to measure thickness of classic CNV lesions. Generally, SD-OCT is an accurate means of assessing CNV lesion area, volume, and retinal thickness. However, this relies on adequate quality of OCT B-scan images. We found that in the few cases where SD-OCT automatic segmentation differed from manual segmentation, it was due to poorer B-scan images. This may be due to patient factors such as a poorly dilated pupil or poor fixation. Another limitation of OCT technology is how it deals with areas of decreased brightness, which may represent fluid but does not necessarily correlate with the presence of nAMD. While SD-OCT automatic segmentation is reliable for classic CNV lesions, this assumes a classic half-ellipsoid shape of the lesion. The inability to process deviations from this shape is one limitation of the SD-OCT analysis software.

Despite these limitations, SD-OCT remains a useful and robust tool for assessing classic CNV lesion size. OCT cannot replace fundus FA to diagnose $\mathrm{CNV}$ as the sensitivity and specificity needs to be equivalent. ${ }^{33}$ However, by comparing serial B-scan images of patient's before and after three anti-VEGF injections, clinicians can monitor disease regression in a non-invasive manner and personalize injection frequency according to patient's visual needs. We anticipate that this approach to monitoring disease progression can be applied to non-classic CNV lesions in nAMD. OCT software will always be prone to segmentation errors and manual correction of automated measurements usually would refine the outputs produced. ${ }^{34,35}$ This study shows that there is good correlation between automated and manual corrected parameters. CNV Lesion size may become a biomarker for treatment response, however further optimization may be essential and necessary with devices such as OCT Angiography. Better and faster software algorithms may achieve even more accurate and predictable measures of disease regression on treatment and recurrence of activity to allow clinicians to make management decisions of types of regimes used for injection therapy.

\section{Conclusion}

In conclusion, we report that SD-OCT automatic segmentation using analysis software 1) can qualitatively show size reduction of classic CNV lesions in nAMD patients pre- versus post anti-VEGF treatment; and 2) in this small cohort is an accurate diagnostic tool to measure CNV lesion thickness and calculate surface area and volume as a further biomarker of disease progression and treatment frequency. 
This study has shown that quantification of SHRM using SD-OCT using automated software is reliable as a biomarker of disease onset and its regression and growth can be used in future studies where combination therapy with anti-VEGF and anti-platelet derived growth factor (PDGF) injections are used at variable time points.

\section{Funding}

None.

\section{Acknowledgments}

None.

\section{Conflicts of interest}

The authors declare that there was no conflict of interest.

\section{References}

1. Grossniklaus HE, Gass JD. Clinicopathologic correlations of surgically excised type 1 and type 2 submacular choroidal neovascular membranes. Am J Ophthalmol. 1998;126(1):59-69.

2. Lim LS, Mitchell P, Seddon JM, et al. Age-related macular degeneration. Lancet. 2012;379((9827)):1728-1738.

3. Schmidt-Erfurth U, Kriechbaum K, Oldag A. Three-dimensional angiography of classic and occult lesion types in choroidal neovascularization. Invest Ophthalmol Vis Sci. 2007;48(4):1751-1760.

4. Gass JD, Agarwal A, Lavina AM, et al. Focal inner retinal hemorrhages in patients with drusen:an early sign of occult choroidal neovascularization and chorioretinal anastomosis. Retina. 2003;23(6):741-751.

5. Bressler NM. Age-related macular degeneration is the leading cause of blindness. JAMA. 2004;291(15):1900-1901.

6. Bressler NM, Doan QV, Varma R, et al. Estimated cases of legal blindness and visual impairment avoided using ranibizumab for choroidal neovascularization:non-Hispanic white population in the United States with age-related macular degeneration. Arch Ophthalmol. 2011;129(6):709-717.

7. Gemmy Cheung CM, Li X, Cheng CY, et al Prevalence and risk factors for age-related macular degeneration in Indians:a comparative study in Singapore and India. Am K Ophthalmol. 2013;155(4):764-773.

8. Wong TY, Liew G, Mitchell P. Clinical update:new treatments for agerelated macular degeneration. Lancet. 2007;370 (9583):204-206.

9. Brown DM, Kaiser PK, Michels M, et.al. Ranibizumab versus verteporfin for neovascular age-related macular degeneration. $N$ Engl $J$ Med 2006;355(14):1432-1444.

10. Rosenfeld PJ, Brown DM, Heier JS, et al. Ranibizumab for neovascular age-related macular degeneration. $N$ Engl J Med. 2006;355(14):14191431.

11. Martin DF, Maguire MG, Fine SL, et al. Ranibizumab and bevacizumab for treatment of neovascular age-related macular degeneration:two-year results. Ophthalmology. 2012;119(7):1388-1398.

12. Martin DF, Maguire MG, Ying GS, et al. Ranibizumab and bevacizumab for neovascular age-related macular degeneration. $N$ Engl $\mathrm{J}$ Med. 2011;364(20):1897-1908

13. Hee MR, Baumal CR, Puliafito CA, et al. Optical coherence tomography of age-related macular degeneration and choroidal neovascularization. Ophthalmology. 1996;103(8):1260-1270.
14. Coscas F, Coscas G, Souied E, et al. Optical coherence tomography identification of occult choroidal neovascularization in age-related macular degeneration. Am J Ophthalmol. 2007;144(4):592-599.

15. Eter N, Spaide RF. Comparison of fluorescein angiography and optical coherence tomography for patients with choroidal neovascularization after photodynamic therapy. Retina. 2005;25(6):691-696.

16. Schlanitz FG, Ahlers C, Sacu S, et al. Performance of drusen detection by spectral-domain optical coherence tomography. Retina. 2010;51(12):6715-6721.

17. Sadda SR, Wu Z, Walsh AC, et al. Errors in retinal thickness measurements obtained by optical coherence tomography. Ophthalmology. 2006;113(2):285-293.

18. Ray R, Stinnett SS, Jaffe GJ. Evaluation of image artifact produced by optical coherence tomography of retinal pathology. Am J Ophthalmol. 2005;139(1):18-29.

19. Keane PA, Mand PS, Liakopoulos S, et al. Accuracy of retinal thickness measurements obtained with Cirrus optical coherence tomography. $\mathrm{Br} J$ Ophthalmol. 2009;93(11):1461-1467.

20. Drexler W, Fujimoto JG. State-of-the-art retinal optical coherence tomography. Prog Ret Eye Res. 2008;27(1):45-88.

21. Van Velthoven ME, Faber DJ, Verbraak FD, et al. Recent developments in optical coherence tomography for imaging the retina. Prog Ret Eye Res. 2007;26(1):57-77.

22. Witkin AJ, Vuong LN, Srinivasan VJ, et al. High-speed ultrahigh resolution optical coherence tomography before and after ranibizumab for age-related macular degeneration. Ophthalmology. 2009;116(5):956963.

23. Diniz B, Rodger DC, Chavali VR, et al. Drusen and RPE atrophy automated quantification by optical coherence tomography in an elderly population. Eye (Lond). 2015;29(2):272-279.

24. Gregori G, Wang F, Rodenfeld PJ, et al. Spectral domain optical coherence tomography imaging of drusen in nonexudative age-related macular degeneration. Ophthalmology. 2011;118(7):1373-1379.

25. Nittala M, Ruiz-Garcia H, Sadda SR. Accuracy and reproducibility of automated drusen segmentation in eye with non-neovascular age-related macular degeneration. Invest Ophthalmol Vis Sci. 2012;53(13):83198324 .

26. Yehoshua Z, Garcia Filho CA, Penha FM, et al. Comparison of geographic atrophy measurements from the OCT fundus image and the sub-RPE slab image. Ophthalmic Surg Lasers Imaging Retina. 2013;44(2):127-132.

27. Schmidt-Erfurth U, Leitgeb RA, Michels S, et al. Three dimensional ultrahigh-resolution optical coherence tomography of macular diseases. Invest Ophthalmol Vis Sci. 2005;46(9):3393-3402.

28. Stopa M, Bower BA, Davies E, et al. Correlation of pathologic features in spectral domain optical coherence tomography with conventional retinal studies. Retina. 2008;28(2):298-308.

29. Ahlers C, Schmidt-Erfurth U. Three-dimensional high resolution OCT imaging of macular pathology. Opt Express. 2009;17(5):4037-4045.

30. Ishikawa H, Stein DM, Wollstein G, et al. Macular segmentation with optical coherence tomography. Invest Ophthalmol Vis Sci. 2005;46(6):2012-2017.

31. Baroni M, Fortunato P, Torre AL. Towards quantitative analysis of retinal features in optical coherence tomography. Med Engin Phys. 2007;29(4):432-441. 
32. Fabritius T, Makita S, Miura M, et al. Automated segmentation of the macula by optical coherence tomography. Opt Express. 2009;17(18):15659-15669.

33. Amoaku WM, Chakravarthy U, Gale R, et al. Defining response to antiVEGF therapies in neovascular AMD. Eye. 2015;29(6):721-731.
34. Sadda SR, Wu Z, Walsh AC, et al. Errors in retinal thickness measurements obtained by optical coherence tomography. Ophthalmology. 2006;113(2):285-293.

35. Ray R, Stinnett SS, Jaffe GJ. Evaluation of image artifact produced by optical coherence tomography of retinal pathology. Am J Ophthalmol. 2005;139(1):18-29. 